\title{
Microcephalic primordial dwarfism, Alazami type
}

INSERM

\section{Source}

INSERM. (1999). Orphanet: an online rare disease and orphan drug data base.

Microcephalic primordial dwarfism, Alazami type. ORPHA:319671

Microcephalic primordial dwarfism, Alazami type is a rare, genetic developmental defect during embryogenesis syndrome characterized by severe intellectual disability, distinct dysmorphic facial features (i.e. triangular face with prominent forehead, narrow palpebral fissures, deep-set eyes, low-set ears, broad nose, malar hypoplasia, short philtrum, macrostomia, widely spaced teeth) and pre and postnatal proportionate short stature, ranging from primordial dwarfism (height below -3.5 SD) to a milder phenotype with less severe growth restriction (height below -2.5 SD). Other reported features include skeletal findings (e.g. scoliosis), microcephaly, involuntary hand movements, hypersensitivity to stimuli and behavioral problems, such as anxiety. 